\title{
Soluble lectin-like oxidized low density lipoprotein receptor-1 in metabolic syndrome
}

\author{
Palakorn Puttaruk ${ }^{\mathrm{a}}$, Duangnate Pipatsatitpong ${ }^{\mathrm{b}}$, Pilaiwan Siripurkpong \\ ${ }^{a}$ Department of Medical Technology Laboratory, Thammasat University Hospital, Pathum Thani 12120, \\ Thailand \\ ${ }^{b}$ Department of Medical Technology, Faculty of Allied Health Sciences, Thammasat University, Pathum \\ Thani, 12120,Thailand
}

\begin{abstract}
Background: Serum levels of soluble lectin-like oxidized low-density lipoprotein receptor-1 (sLOX-1) reflect increases in LOX-1 receptor expression associated with inflammation and metabolic disorders.

Objectives: To examine sLOX-1 levels in metabolic syndrome and association of sLOX-1 with classical risk factors, and with metabolic syndrome, a clustering of metabolic disorders associated with cardiovascular risk factors.

Methods: We selected 148 serum samples from patient participants with metabolic syndrome and 206 samples from patients with non-metabolic syndrome as controls, using the modified National Cholesterol Educational Program Adult Treatment Panel III (NCEP-ATP III) criteria.

Results: Levels of sLOX-1 were increased significantly in participants with metabolic syndrome $(P<0.001)$. Serum sLOX-1 was positively associated with body mass index (BMI), blood pressure, fasting plasma glucose, triglyceride, and total cholesterol, but negatively associated with high-density lipoprotein cholesterol. Analysis of serum sLOX-1 for metabolic syndrome showed $99.03 \%$ specificity and $100 \%$ sensitivity. The area under the receiver operating characteristic curve was 0.998 (95\%CI 0.996-1.001, $P<0.001)$. A univariate analysis showed sLOX-1 was significantly correlated with metabolic syndrome, but was not after adjustment for sex, age, blood pressure, and BMI. Multivariate regression analysis found that being overweight (82.3; 95\%CI 10.7-631.9), hyperglycemia (1.1; 95\%CI 1.1-1.2), and hypertriglyceridemia (1.1; 95\%CI 1.0-1.1) were significantly correlated with metabolic syndrome. HDL cholesterol was a protective factor (0.96; 95\%CI: 0.93-0.99).

Conclusions: Serum sLOX-1 is a suitable biomarker for diagnosis of metabolic syndrome. However, univariate and multivariate analysis suggested that sLOX-1 may be a modulating factor, and not an independent risk factor.
\end{abstract}

Keywords: Body mass index, fasting plasma glucose, HDL cholesterol, metabolic syndrome, NCEP-ATP III, soluble lectin-like oxidized LDL receptor-1, triglyceride

Metabolic syndrome is a multiplex risk factor for coronary heart disease, diabetes, and fatty liver, and its pathogenesis involves low grade chronic inflammation. The syndrome is a cooccurrence (clustering) of medical conditions including: abdominal (central) obesity, hypertension, hyperglycemia, increased triglyceride, and decreased high density lipoprotein-cholesterol (HDL-cholesterol) levels [1]. Criteria for diagnosis of metabolic syndromes have been defined. The World Health Organization

Correspondence to: Pilaiwan Siripurkpong, Department of Medical Technology, Faculty of Allied Health Sciences, Thammasat University, Pathum Thani 12120,Thailand.

E-mail: spilaiwa@tu.ac.th
(WHO) in 1999 determined insulin resistance as pivotal for diagnosis of metabolic syndrome [2], while the National Cholesterol and Education Program Adult Treatment Panel III (NCEP-ATP III), American Association of Clinical Endocrinology, and International Diabetes Federation (IDF) adopted abdominal obesity as pivotal [3-6]. However, the core components of the metabolic syndrome, which include dyslipidemia, impaired glucose tolerance, increased waist circumference and hypertension, are classical risk factors required for diagnosis [7].

The lectin-like oxidized low density lipoprotein receptor (LOX-1) is a receptor for oxidized low density lipoprotein (oxidized LDL) and has been implicated in vascular inflammation, and atherosclerotic plague 
formation and destabilization [8, 9]. Binding of oxidized LDL to LOX-1 on vascular endothelial cells leads to the activation of nuclear factor- $\mathrm{kb}$, resulting in endothelial cell dysfunction and increases in monocyte adhesion [10]. Proinflammatory and oxidative stimuli during atherogenesis, and advanced atherosclerotic plaques prominently upregulate LOX-1 expression $[8,11,12]$. LOX-1 is a type II membrane protein containing 4 domains including: a short $\mathrm{N}$-terminal cytoplasmic domain, a transmembrane domain, a neck domain, and a lectin-like extracellular C-terminal domain $[13,14]$. The extracellular domain can be cleaved proteolytically and released as a soluble form (sLOX-1). An elevated circulating level of sLOX-1 may reflect increases in LOX-1 receptor expression, inflammation, and metabolic disorder [15-17]. sLOX1 is elevated in the plasma of individuals with obesity [18] and diabetic mellitus [19], which are characterized as medical conditions of metabolic syndrome.

Plasma level of sLOX-1 has been proposed as a potential marker for chronic vascular inflammation. Therefore, the aim of this study was to verify the serum level of sLOX-1 in metabolic syndrome. The correlation of sLOX-1 and other classical risk factors for metabolic syndrome was also validated.

\section{Materials and methods \\ Study population and study design}

A case-control study was conducted at Thammasat University Hospital, Thailand. A total of 1,100 participants, Thammasat University officers who presented for an annual checkup in January 2009, were retrospectively enrolled and their demographic data (sex, age, weight, height, and blood pressure) were recorded. We selected 148 cases of metabolic syndrome and 206 normal controls using the National Cholesterol Educational Program Adult Treatment Panel III (NCEP-ATP III) criteria with a modification, replacing waist circumference with body mass index (BMI) [20, 21]. According to the modified NCEPATP III guidelines, metabolic syndrome is diagnosed when three or more of the following risk factors are present: (1) BMI $\geq 25 \mathrm{~kg} / \mathrm{m}^{2}$ for women and $\geq 27 \mathrm{~kg} /$ $\mathrm{m}^{2}$ for men, (2) fasting plasma glucose (FPG) $\geq 110$ $\mathrm{mg} / \mathrm{dL}$, (3) systolic blood pressure $\geq 130 \mathrm{mmHg}$ or diastolic blood pressure $\geq 85 \mathrm{mmHg}$, (4) fasting triglyceride $\geq 150 \mathrm{mg} / \mathrm{dL}$, and (5) high-density lipoprotein cholesterol (HDL cholesterol) $<40 \mathrm{mg} / \mathrm{dL}$ in men and $<50 \mathrm{mg} / \mathrm{dL}$ in women. For control participants without metabolic syndrome, none above five risk factors were presented. The research protocol was reviewed and approved by the Human Research Ethics Committee of Thammasat University (No. 40/52). The Ethics Committee specifically waived the usual requirement for informed consent provided patient confidentiality was protected according to the principles of the contemporary version of the Declaration of Helsinki, and permitted demographic data collection and the reuse of samples taken for clinical purposes at the annual checkup in January, 2009.

\section{Blood collection and measurement of classical risk factors}

Fasting blood samples of all participants were drawn and used for determining the classical risk factors: FPG, total cholesterol, HDL-cholesterol, LDLcholesterol, and triglyceride using automated blood analyzer (Dimension clinical chemistry; RxL Max, Siemens).

\section{Measurement of sLOX-1}

Serum sLOX-1 was analyzed using sandwich enzyme-linked immunosorbent assay (DY1798, R\&D system) and ELISA plate reader (Opsys MR, Dynex). Briefly, in each well of the microplate mouse was coated with anti-human LOX-1 (2 $\mu \mathrm{g} / \mathrm{mL})$. After blocking nonspecific binding sites with $1 \%$ bovine serum albumin in phosphate-buffered saline (PBS) diluent, test sample or standard (recombinant human LOX-1) dissolved in diluent (31-2000 pg/mL) were added ( $0.1 \mathrm{~mL}$ per well). After incubation for 2 hours at room temperature, the well was washed with 0.05\% Tween 20 in PBS, pH 7.4. The sLOX-1 and recombinant human LOX-1 retained on the wells were detected by adding biotinylated goat anti-human LOX-1, streptavidin conjugated to horseradishperoxidase, and tetramethyl benzidine substrate. The color reaction was determined using microplate reader at $\lambda_{450 \mathrm{~nm}}$, subtracted with the reading at $\lambda_{570 \mathrm{~nm}}$. The coefficient of variation was $3.6 \%(\mathrm{n}=10)$ and the percent recovery was103.3\% $(n=10)$. Sensitivity and specificity of sLOX-1 were analyzed using a receiver operating characteristic (ROC) curve.

\section{Statistical analyses}

Results are expressed as means and standard deviation (SD) or as median and interquartile range if the distribution of the data is skewed. Comparisons were performed using a chi-square or Fisher exact 
test for proportion, and a Student $t$ or Mann-Whiney $U$ test for continuous variables. The level of significance was set at $P<0.05$. Spearman's rank correlation coefficients were used to examine the correlation between sLOX-1 levels and age, BMI, blood pressure, FPG, and lipid profiles with a significance cut off at $P<0.001$. Multiple linear regression analysis was used to evaluate the contribution of each confounding factor for SLOX-1. Univariate and multivariate analysis were conducted using a logistic regression with 95\% confidence interval. The Statistical Package for the Social Sciences software (version 16, SPSS, Chicago, IL, USA) was used for statistical analyses.

\section{Results \\ Study population}

We enrolled 148 participants with metabolic syndrome and 206 controls, and their characteristics are summarized in Table $\mathbf{1 .}$

The average age of participants with metabolic syndrome was significantly more than that of the controls, but there were not significantly more women or men in either group. BMI, blood pressure, fasting plasma glucose, total cholesterol, HDL cholesterol, and triglyceride were all significantly different between the metabolic syndrome and the control groups. Most characteristics in metabolic cases, with the exception of HDL-cholesterol, had higher average values than in the controls. However, LDL cholesterol levels were similar in both groups.

\section{Serum sLOX-1 in metabolic syndrome}

Serum sLOX-1 levels were higher in participants with metabolic syndrome [166.5 (136.5-182.1) pg/ $\mathrm{mL}$ ] than in controls [63.3 (50.3-71.3) $\mathrm{pg} / \mathrm{mL}$, $P<0.001$ (Figure 1A) with no significant differences between the sexes in either metabolic syndrome [161.05 (135.92-179.33) $\mathrm{pg} / \mathrm{mL}$ in men vs 169.75 (136.42-191.25) $\mathrm{pg} / \mathrm{mL}$ in women, $P=0.449$ ] or control groups [58.14 (47.76-69.72) pg/mL in men vs 63.96 (51.10-72.21) $\mathrm{pg} / \mathrm{mL}$ in women, $P=0.503)]$ (Figure 1B).

Correlations of sLOX-1 levels with classical metabolic risk factors and serum lipid profiles were analyzed and the results are shown in Table 2 .

The circulating sLOX-1 levels were significantly correlated with age, BMI, blood pressure (BP), both systolic and diastolic. They were also correlated with FPG, total cholesterol, triglyceride, and HDL cholesterol, but not significantly correlated with sex or LDL cholesterol. Correlation between sLOX-1 and risk factors in Table 2 was analyzed by multiple linear regression and the results are shown in Table 3.

Table 1. Characteristics of all enrolled participants

\begin{tabular}{|c|c|c|c|}
\hline Characteristics & $\begin{array}{l}\text { Metabolic syndrome } \\
(n=148)\end{array}$ & $\begin{array}{l}\text { Control with non-metabolic } \\
\text { syndrome }(n=206)\end{array}$ & $\boldsymbol{P}$ \\
\hline \multicolumn{4}{|l|}{ Sex } \\
\hline Female, n (\%) & $87(59)$ & $131(64)$ & \\
\hline Male, n (\%) & $61(41)$ & $75(36)$ & $0.36^{\mathrm{a}}$ \\
\hline Age (years) & $51.6(11.7)$ & $44(11.9)$ & $<0.001^{* b}$ \\
\hline Body mass index $\left(\mathrm{kg} / \mathrm{m}^{2}\right)$ & $27.9(25.8-30.1)$ & $21.7(19.923 .5)$ & $<0.001^{* c}$ \\
\hline \multicolumn{4}{|l|}{ Blood pressure (BP) (mmHg) } \\
\hline Systolic BP & 139(132.0-150.0) & $110(105.0-115.0)$ & $<0.001^{* c}$ \\
\hline Diastolic BP & $84(77.3-91.0)$ & $70(64.8-73.0)$ & $<0.001^{* c}$ \\
\hline Fasting plasma glucose (mg/dL) & $101(89.0-125.8)$ & 85.5(81.8-90.0) & $<0.001^{* c}$ \\
\hline Total cholesterol (mg/dL) & $226.4(49.2)$ & $205.9(40.5)$ & $<0.001^{* b}$ \\
\hline HDL cholesterol (mg/dL) & $45(37.0-53.8)$ & $62(53.074 .0)$ & $<0.001^{* c}$ \\
\hline LDL cholesterol (mg/dL) & $118.5(45.8)$ & $118.8(35.8)$ & $0.94^{\mathrm{b}}$ \\
\hline Triglyceride (mg/dL) & $192(155.0-238.8)$ & $69(52.895 .3)$ & $<0.001^{* c}$ \\
\hline
\end{tabular}

HDL = high-density lipoprotein, LDL = low-density lipoprotein. Continuous variables are shown as means (standard deviation) or median (interquartile range). Categorical variables are shown as percentage (\%). ${ }^{\mathrm{a}}$ Chi-square test, ${ }^{\mathrm{b}}$ Student $t$ test, ${ }^{\mathrm{c}}$ Mann-Whitney $U$ test, ${ }^{*} P<0.05$. 

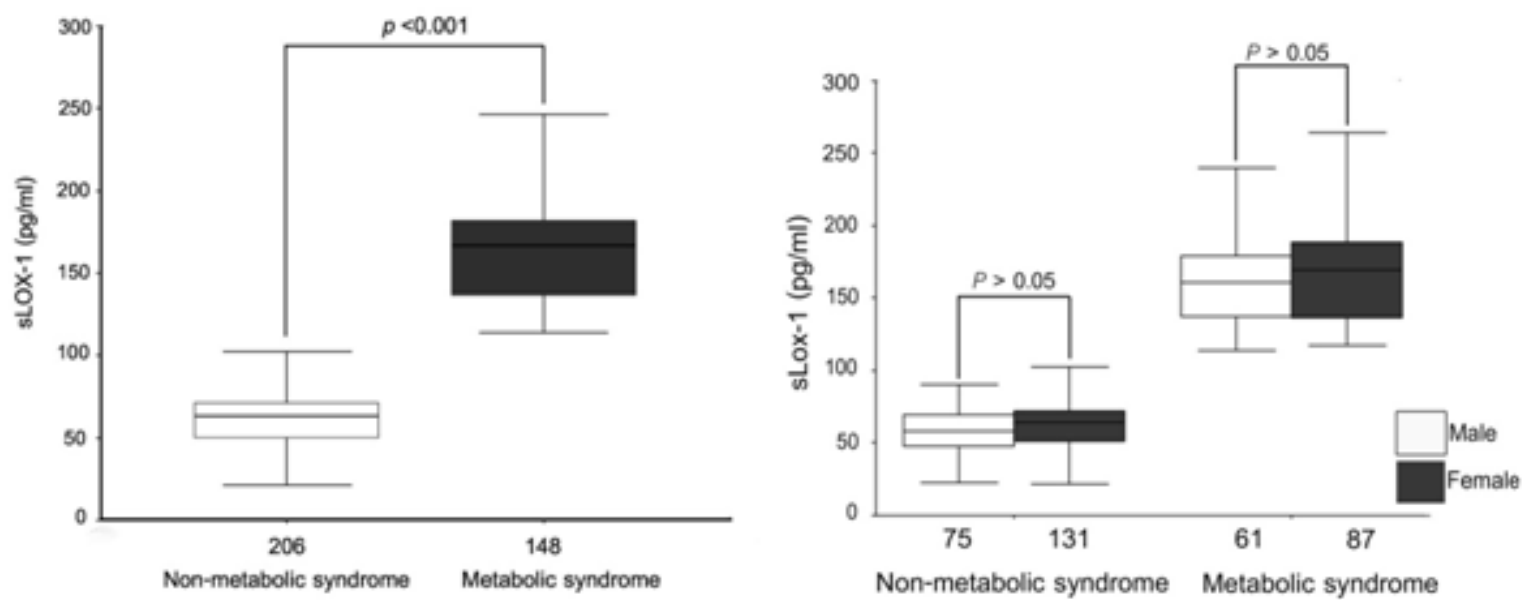

Figure 1. Serum levels of sLOX-1 in metabolic syndrome. A (left) Comparison of the sLOX-1 levels in metabolic syndrome and control with non-metabolic syndrome, and B (right) Comparison of the sLOX-1 levels in male (open bars) and female (solid bars) separately in both groups.

Table 2. Correlation of soluble lectin-like oxidized low density lipoprotein receptor-1 with age, sex, body mass index, blood pressure, fasting plasma glucose and lipid profiles.

\begin{tabular}{lll}
\hline Characteristic & Correlation coefficient $(\boldsymbol{r})$ & $\boldsymbol{P}$ \\
\hline Age & 0.29 & $<0.001^{*}$ \\
Sex & -0.002 & 0.997 \\
Body mass index & 0.62 & $<0.001^{*}$ \\
Blood Pressure (BP) $\quad$ & $<0.001^{*}$ \\
$\quad$ Systolic BP & 0.63 & $<0.001^{*}$ \\
$\quad$ Diastolic BP & 0.48 & $<0.001^{*}$ \\
Fasting plasma glucose & 0.495 & $0.001^{*}$ \\
Total cholesterol & 0.177 & $<0.001^{*}$ \\
Triglyceride & 0.633 & $<0.001^{*}$ \\
High-density lipoprotein cholesterol & -0.384 & 0.46 \\
Low-density lipoprotein cholesterol & -0.041 & \\
\hline
\end{tabular}

Spearman's rank $(P<0.001)$

Table 3. Multiple linear regression analysis of soluble lectin-like oxidized low density lipoprotein receptor-1 and age, blood pressure (BP), body mass index (BMI), fasting plasma glucose (FPG), total cholesterol, triglyceride, and high-density lipoprotein (HDL) cholesterol

\begin{tabular}{|c|c|c|c|c|c|}
\hline \multirow[t]{2}{*}{ Independent factors } & \multicolumn{2}{|c|}{ Unstandardized coefficients } & \multirow{2}{*}{$\begin{array}{c}\text { Standardized coefficients } \\
\beta\end{array}$} & \multirow[t]{2}{*}{$t$} & \multirow[t]{2}{*}{$(P<0.05)$} \\
\hline & $\beta$ & Std. Error & & & \\
\hline Constant & -140.1 & 19.9 & & -7.05 & $<0.001$ \\
\hline Age & 0.41 & 0.19 & 0.09 & 2.21 & 0.03 \\
\hline Systolic BP & 1.06 & 0.20 & 0.35 & 5.26 & $<0.001$ \\
\hline Diastolic BP & -0.17 & 0.29 & -0.03 & -0.58 & 0.56 \\
\hline Body mass index & 4.07 & 0.63 & 0.30 & 6.43 & $<0.001$ \\
\hline Fasting plasma glucose & 0.21 & 0.08 & 0.11 & 2.58 & 0.01 \\
\hline Total cholesterol & -0.07 & 0.05 & -0.06 & -1.44 & 0.15 \\
\hline Triglyceride & 0.08 & 0.02 & 0.198 & 4.39 & $<0.001$ \\
\hline HDL cholesterol & -0.14 & 0.09 & -0.06 & -1.48 & 0.14 \\
\hline
\end{tabular}

sLOX-1 =-140.07 + 0.41 (age) + 1.06 (SBP) + 4.32 (BMI) + 0.19 (FPG) + 0.07 (triglyceride)

$R=0.76$ and $R^{2}=0.57$ 
Results showed that age, SBP, BMI, FPG, and triglyceride were independently and significantly associated with sLOX-1 level.

Sensitivity and specificity of sLOX-1 were studied. At the cut-off value (114 pg/mL), sLOX-1 showed $99.0 \%$ vs $98.7 \%$ specificity and $100 \%$ vs $100 \%$ sensitivity in diagnosis of metabolic syndrome and control, respectively. The ROC curves presented a true positive fraction (sensitivity as $\mathrm{Y}$ axis) plotted against the false positive fraction ( 1 - specificity as $\mathrm{X}$ axis) for the diagnosis of metabolic syndrome. Area under the ROC curve was 0.998 (95\%CI: 0.9961.001, $P<0.001$ ), indicating that the level of sLOX-1 was highly sensitive and specific for metabolic syndrome.

\section{Serum SLOX-1 as risk factor in metabolic syndrome}

The univariate and multivariate logistic regression analyses of several parameters for predictive index of metabolic syndrome are summarized in Table 4.
Univariate regression found that age older than 36 years, being overweight (BMI $\geq 25 \mathrm{~kg} / \mathrm{m}^{2}$ for women and $\geq 27 \mathrm{~kg} / \mathrm{m}^{2}$ for men), hypertension (SBP $\geq 130$ $\mathrm{mmHg}$, DBP $\geq 85 \mathrm{mmHg}$ ), hyperglycemia (FBG $\geq 110$ $\mathrm{mg} / \mathrm{dL}$ ), hypertriglyceridemia ( $\geq 150 \mathrm{mg} / \mathrm{dL}$ ), hypercholesterolemia ( $\geq 200 \mathrm{mg} / \mathrm{dL}$ ), low HDL cholesterol $(<40 \mathrm{mg} / \mathrm{dL}$ in men and $<50 \mathrm{mg} / \mathrm{dL}$ in women), and high sLOX-1 level (>114 pg/mL) are significantly associated with metabolic syndrome.

Multivariate regression analysis found that being overweight, hyperglycemia, and hypertriglyceridemia produced an 82.3- (95\%CI 10.7-631.9), 1.1- (95\%CI 1.1-1.2), and 1.1- (95\%CI 1.0-1.1)-fold higher risk of having metabolic syndrome, after adjusting for sex, age, SBP, DBP, BMI, and other variables (Table 4). HDL cholesterol level indicated a protective factor for metabolic syndrome of 0.96 (95\%CI: 0.93-0.99).

Table 4. Univariate and multivariate analyses of risk factors for metabolic disorder

\begin{tabular}{|c|c|c|c|c|c|c|}
\hline Variables & $\begin{array}{l}\text { Case } \\
\text { n (\%) }\end{array}$ & $\begin{array}{l}\text { Control } \\
\text { n (\%) }\end{array}$ & $\begin{array}{l}\text { Crude OR } \\
(95 \% \text { CI })\end{array}$ & $\boldsymbol{P}$ & $\begin{array}{l}\text { Adjusted OR } \\
(95 \% \mathrm{CI})\end{array}$ & $\boldsymbol{P}$ \\
\hline \multicolumn{7}{|l|}{ Sex } \\
\hline Male & $61(41)$ & $75(36)$ & 1 & & & \\
\hline Female & $87(59)$ & $131(64)$ & $0.8(0.5-1.3)$ & 0.36 & & \\
\hline \multicolumn{7}{|l|}{ Age (years) } \\
\hline $25-35$ & $17(12)$ & $60(29)$ & 1 & 0.24 & 1 & \\
\hline $36-45$ & $26(18)$ & $60(29)$ & $1.5(0.8-3.1)$ & $<0.001^{*}$ & $0.1(0.004-2.9)$ & 0.181 \\
\hline $46-55$ & $48(32)$ & $50(24)$ & $3.4(1.7-6.6)$ & $<0.001 *$ & $0.9(0.1-9.4)$ & 0.942 \\
\hline$>55$ & $57(38)$ & $36(18)$ & $5.6(2.8-11.0)$ & $<0.001^{*}$ & $2.1(0.2-20.5)$ & 0.518 \\
\hline Body mass index $\left(\mathrm{kg} / \mathrm{m}^{2}\right)$ & & & & & 1 & \\
\hline Normal & $30(20)$ & 195(95) & 1 & & 82.3 & $<0.001^{*}$ \\
\hline Over weight & $118(80)$ & $11(5)$ & $69.7(33.7-144.4)$ & $<0.001^{*}$ & $(10.7-631.9)$ & \\
\hline Systolic BP (mmHg) & $148(100)$ & $206(100)$ & $1.2(1.2-1.3)$ & $<0.001^{*}$ & & \\
\hline Diastolic BP (mmHg) & $148(100)$ & $206(100)$ & $1.2(1.1-1.2)$ & $<0.001^{*}$ & & \\
\hline $\mathrm{FPG}(\mathrm{mg} / \mathrm{dL})$ & $148(100)$ & $206(100)$ & $1.1(1.1-1.2)$ & $<0.001^{*}$ & $1.1(1.1-1.2)$ & $<0.001^{*}$ \\
\hline Total cholesterol (mg/dL) & $148(100)$ & $206(100)$ & $1.01(1.00-1.02)$ & $<0.001 *$ & & \\
\hline Triglyceride (mg/dL) & $148(100)$ & $206(100)$ & $1.1(1.0-1.1)$ & $<0.001^{*}$ & $1.1(1.0-1.1)$ & $<0.001^{*}$ \\
\hline HDL cholesterol (mg/dL) & $148(100)$ & $206(100)$ & $0.96(0.95-0.98)$ & $<0.001 *$ & $0.96(0.93-0.99)$ & $0.002 *$ \\
\hline LDL cholesterol (mg/dL) & $148(100)$ & $206(100)$ & $1.00(0.99-1.01)$ & 0.937 & & \\
\hline sLOX-1 (pg/mL) & $148(100)$ & $206(100)$ & $1.2(1.1-1.4)$ & $<0.001^{*}$ & & \\
\hline
\end{tabular}

$\mathrm{OR}=$ odds ratio, $\mathrm{CI}=$ confidence interval, $\mathrm{FPG}=$ fasting plasma glucose, $\mathrm{BP}=$ blood pressure, $\mathrm{HDL}=$ high-density lipoprotein, LDL = low-density lipoprotein. Data were adjusted for sex, age, systolic BP, diastolic BP, body mass index, and other variables in the model. $* P<0.05$ 


\section{Discussion}

Metabolism is a vital cellular chemical process. Disorders of metabolism lead to a group of risk factors for heart disease and other health problems [22]. It has been shown that patients with metabolic syndrome are at high-risk of developing many disorders. The sLOX-1 levels have been shown to significantly increase in atherosclerotic plaque instability [23], diabetes mellitus [19], obesity [18], and are positively correlated with a reduction in body weight [24]. Association with coronary artery disease and metabolic disorders also suggested sLOX-1 as a biomarker for early detection of acute coronary syndrome $[25,26]$. We therefore sought to assess whether serum sLOX-1 levels could be used as an index of metabolic syndrome.

Determination of serum sLOX-1 levels indicated an elevation in patients with metabolic syndrome compared with controls $(P<0.001)$. The result was consistent with that reported by Civelek et al. [27], but the number of participants with metabolic syndrome was higher in our study. The results also showed that sLOX-1 levels were positively correlated with blood pressure, BMI, FPG, and triglyceride, but negatively correlated with HDL cholesterol (Table 2), and serum sLOX-1 level depended on independent factors: age, SBP, BMI, FPG, and triglyceride (Table 3). Correlation between serum sLOX-1 levels and main criteria for diagnosis of metabolic syndrome suggested serum sLOX-1 level as a diagnostic index of metabolic syndrome. However, no correlation was observed between sLOX-1and LDL cholesterol ( $\rho=-0.02, P=0.827)$. This finding is consistent with previous studies showing no significant correlation (Spearman's $\rho=-0.02$; $P=0.827$ ) [25].

Level of serum sLOX-1 at cut-off value (114 pg/ $\mathrm{mL}$ ) showed efficient ability to differentiate metabolic syndrome from normal controls with sensitivity and specificity of $100 \%$ and $99 \%$, respectively. The large area under the ROC curve (0.998) suggested that sLOX-1 is a suitable single biomarker with which to diagnose metabolic syndrome. However, given the modified NCEP-ATP III criteria, participants in the control group in the present study were identified as showing normal for all variables. Therefore, specificity of sLOX-1 in this study was higher than that previously reported (41.4\%) [27]. The difference in average ages of participants with metabolic syndrome and controls was a limitation of this study. Because prevalence of metabolic syndrome increased strongly with age [28], a higher number of participants with older age were enrolled in the metabolic syndrome group.

The univariate analysis found that serum sLOX1 was strongly correlated with metabolic syndrome as shown in Table 4. However, after adjustment for sex, age, blood pressure, and BMI, the associations between sLOX-1 and metabolic syndrome did not reach statistical significance. Therefore, sLOX-1 was not considered an independent risk factor for metabolic syndrome. Multivariate analysis found that BMI, FPG, systolic blood pressure, and triglyceride significantly correlated with metabolic syndrome in agreement with the criteria classified by NCEP-ATP III.

Previous study has shown that sLOX-1 increases markedly in acute coronary syndrome (ACS). Level of sLOX-1 was a more specific and sensitive biomarker of ACS than others such as cardiac troponin $\mathrm{T}$ (cTnT), heart type-fatty acid binding protein, and high sensitive C-reactive protein [26, 29]. The sLOX-1 was recommended as a suitable marker for early diagnosis of ACS than cTnT because 86\% of the cTnT-negative patients showed sLOX-1 levels $>1.0 \mathrm{ng} / \mathrm{mL}$ serum. Serum sLOX-1 levels were suggested to rise before ACS onset [25]. Taking together the previous results and the elevation of serum sLOX-1 levels associated with metabolic syndrome in the present study, serum sLOX-1 level might be used to predict the occurrence of cardiovascular events in patients with metabolic syndrome. The increased levels of sLOX-1 in people with metabolic syndrome suggest an increased risk for cardiovascular events. In further study, the cutoff value of sLOX-1 level should be considered for predicting ACS in patients with metabolic syndrome.

\section{Conclusion}

Serum sLOX-1 increased in metabolic syndrome with high sensitivity and specificity for discriminating metabolic syndrome from non-metabolic syndrome. Therefore, sLOX-1 is potentially a suitable diagnostic marker of metabolic syndrome. However, univariate and multivariate analysis suggested that sLOX-1 might be a modulating, not independent, risk factor for metabolic syndrome.

\section{Acknowledgments}

We thank Assistant Professor Sudawadee Kongkhum for critical reading of the manuscript. 
We also acknowledge the Department of Medical Technology, Faculty of Allied Health Sciences, Thammasat University for providing all research facilities. This work was financially supported by Thammasat University Research Fund.

\section{Conflicts of interest statement}

The authors have no conflict of interest to declare.

\section{References}

1. Grundy SM, Brewer HB, Jr, Cleeman JI, Smith SC, Jr, Lenfant C, et al. for the American Heart Association. Definition of metabolic syndrome: report of the National Heart, Lung, and Blood Institute/American Heart Association conference on scientific issues related to definition. Circulation. 2004; 109:433-8.

2. Balkau B, Charles MA. Comment on the provisional report from the WHO consultation. European Group for the Study of Insulin Resistance (EGIR). Diabet Med. 1999; 16:442-3.

3. Genuth S, Alberti KG, Bennett P, Buse J, Defronzo R, Kahn R, et al. Follow-up report on the diagnosis of diabetes mellitus. Diabetes Care. 2003; 26:3160-7.

4. Expert Panel on Detection, Evaluation, and Treatment of High Blood Cholesterol in Adults. Executive summary of the third report of the National Cholesterol Education Program (NCEP) expert panel on detection, evaluation, and treatment of high blood cholesterol in adults (adult treatment panel III). JAMA. 2001; 285: 2486-97.

5. Sirdah MM, Abu Ghali AS, Al Laham NA. The reliability of the National Cholesterol Education Program's Adult Treatment Panel III (NCEP/ATP III) and the International Diabetes Federation (IDF) definitions in diagnosing metabolic syndrome (MetS) among Gaza Strip Palestinians. Diabetes Metab Syndr. 2012; 6:4-8.

6. Rezaianzadeh A, Namayandeh SM, Sadr SM. National cholesterol education program adult treatment panel III vs international diabetic federation definition of metabolic syndrome, which one is associated with diabetes mellitus and coronary artery disease? Int $\mathrm{J}$ Prev Med. 2012; 3:552-8.

7. Eapen D, Kalra GL, Merchant N, Arora A, Khan BV. Metabolic syndrome and cardiovascular disease in South Asians. Vasc Health Risk Manag. 2009; 5: 731-43.

8. Kuge Y, Kume N, Ishino S, Takai N, Ogawa Y, Mukai T, et al. Prominent lectin-like oxidized low density lipoprotein (LDL) receptor-1 (LOX-1) expression in atherosclerotic lesions is associated with tissue factor expression and apoptosis in hypercholesterolemic rabbits. Biol Pharm Bull. 2008; 31:1475-82.

9. Kataoka H, Kume N, Miyamoto S, Minami M, Moriwaki $\mathrm{H}$, Murase T, et al. Expression of lectinlike oxidized low-density lipoprotein receptor-1 in human atherosclerotic lesions. Circulation. 1999; 99:3110-7.

10. Dunn S, Vohra RS, Murphy JE, Homer-Vanniasinkam S, Walker JH, Ponnambalam S. The lectin-like oxidized low-density-lipoprotein receptor: a pro-inflammatory factor in vascular disease. Biochem J. 2008; 409: 349-55.

11. Ishino S, Mukai T, Kume N, Asano D, Ogawa M, Kuge $\mathrm{Y}$, et al. Lectin-like oxidized LDL receptor-1 (LOX-1) expression is associated with atherosclerotic plaque instability -analysis in hypercholesterolemic rabbits. Atherosclerosis. 2007; 195:48-56.

12. Pirillo A, Catapano AL. Soluble lectin-like oxidized low density lipoprotein receptor-1 as a biochemical marker for atherosclerosis-related diseases. Dis Markers. 2013; 35:413-8.

13. Xie Q, Matsunaga S, Niimi S, Ogawa S, Tokuyasu K, Sakakibara Y, et al. Human lectin-like oxidized lowdensity lipoprotein receptor-1 functions as a dimer in living cells. DNA Cell Biol. 2004; 23:111-7.

14. Aoyama T, Sawamura T, Furutani Y, Matsuoka R, Yoshida MC, Fujiwara H, et al. Structure and chromosomal assignment of the human lectin-like oxidized low-density-lipoprotein receptor-1 (LOX-1) gene. Biochem J. 1999; 339:177-84.

15. Navarra T, Del Turco S, Berti S, Basta G. The lectinlike oxidized low-density lipoprotein receptor-1 and its soluble form: cardiovascular implications. J Atheroscler Thromb. 2010; 17:317-31.

16. Murase T, Kume N, Kataoka H, Minami M, Sawamura T, Masaki T, et al. Identification of soluble forms of lectin-like oxidized LDL receptor-1. Arterioscler Thromb Vasc Biol. 2000; 20:715-20.

17. Brinkley TE, Kume N, Mitsuoka H, Brown MD, Phares DA, Ferrell RE, et al. Variation in the human lectin-like oxidized low-density lipoprotein receptor 1 (LOX-1) gene is associated with plasma soluble LOX-1 levels. Exp Physiol. 2008; 93:1085-90.

18. Brinkley TE, Kume N, Mitsuoka H, Phares DA, Hagberg JM. Elevated soluble lectin-like oxidized LDL receptor-1 (sLOX-1) levels in obese postmenopausal women. Obesity (Silver Spring). 2008; 16:1454-6.

19. Tan KC, Shiu SW, Wong Y, Leng L, Bucala R. Soluble lectin-like oxidized low density lipoprotein receptor-1 in type 2 diabetes mellitus. J Lipid Res. 2008; 49: 
1438-44

20. Pongchaiyakul C, Nguyen TV, Kosulwat V, Rojroongwasinkul N, Charoenkiatkul S, Pongchaiyakul $\mathrm{C}$, et al. Defining obesity by body mass index in the Thai population: an epidemiologic study. Asia Pac J Clin Nutr. 2006; 15:293-9.

21. Pongchaiyakul C, Nguyen TV, Wanothayaroj E, Karusan N, Klungboonkrong V. Prevalence of metabolic syndrome and its relationship to weight in the Thai population. J Med Assoc Thai. 2007; 90: 459-67.

22. Grundy SM. Metabolic syndrome: a multiplex cardiovascular risk factor. J Clin Endocrinol Metab. 2007; 92:399-404.

23. Kobayashi N, Takano M, Hata N, Kume N, Yamamoto M, Yokoyama S, et al. Soluble lectin-like oxidized LDL receptor-1 (sLOX-1) as a valuable diagnostic marker for rupture of thin-cap fibroatheroma: verification by optical coherence tomography. Int J Cardiol. 2013; 168:3217-23.

24. Nomata Y, Kume N, Sasai H, Katayama Y, Nakata Y, Okura $\mathrm{T}$, et al. Weight reduction can decrease circulating soluble lectin-like oxidized low-density lipoprotein receptor-1 levels in overweight middleaged men. Metabolism. 2009; 58:1209-14.
25. Hayashida K, Kume N, Murase T, Minami M, Nakagawa D, Inada T, et al. Serum soluble lectin-like oxidized low-density lipoprotein receptor-1 levels are elevated in acute coronary syndrome: a novel marker for early diagnosis. Circulation. 2005; 112:812-8.

26. Kume N, Mitsuoka H, Hayashida K, Tanaka M, Kominami G, Kita T. Soluble lectin-like oxidized LDL receptor-1 (sLOX-1) as a sensitive and specific biomarker for acute coronary syndrome-Comparison with other biomarkers. J Cardiol. 2010; 56:159-65.

27. Civelek S, Kutnu M, Uzun H, Erdenen F, Altunoglu E, Andican G, et al. Soluble lectin-like oxidized LDL receptor 1 as a possible mediator of endothelial dysfunction in patients with metabolic syndrome. J Clin Lab Anal. 2015; 29:184-90.

28. Hildrum B, Mykletun A, Hole T, Midthjell K, Dahl AA. Age-specific prevalence of the metabolic syndrome defined by the International Diabetes Federation and the National Cholesterol Education Program: the Norwegian HUNT 2 study. BMC Public Health. 2007; 7:220.

29. Kume N, Mitsuoka H, Hayashida K, Tanaka M, Kita T. Soluble lectin-like oxidized low-density lipoprotein receptor-1 predicts prognosis after acute coronary syndrome-a pilot study. Circ J. 2010; 74:1399-404. 